\title{
Accuracy of recording of deaths from asthma in the UK: the false negative rate
}

\author{
H F Guite, P G J Burney
}

\begin{abstract}
Background - A study was carried out to determine the extent to which asthma deaths are wrongly attributed to another cause on UK death certificates.

Methods - Deaths from all causes occurring anywhere in the UK were identified amongst 2382 subjects aged 16-64 years within three years of discharge following hospital treatment for asthma (ICD9 493) in hospitals in the South East Thames region. The deaths were reviewed by an expert panel to assess the proportion of asthma deaths identified by the panel which were attributed to another cause of death on the death certificate (false negatives).
\end{abstract}

Results - Eighty five deaths from all causes were identified in a mean follow up period of two years and three months. In 61 cases $(72 \%)$ there was sufficient information for the expert panel to be confident about the cause of death. The panel identified 22 deaths from asthma, four of which were certified as non-asthma deaths (two as deaths from chronic obstructive pulmonary disease (COPD) and two as deaths from cardiovascular disease). The proportion of false negative death certificates was four of $22(18 \%, 95 \%$ confidence interval (CI) 5 to 40).

Conclusions - There is evidence that asthma deaths in the UK are wrongly certified as deaths from both chronic obstructive pulmonary disease and diseases of the cardiovascular system.

(Thorax 1996;51:924-928)

King's College School of Medicine and Dentistry, Department of Public Health and Epidemiology, London SE5 9RS, UK H F Guite

Department of Public Health Medicine, UMDS,

St Thomas' Hospital, London SE1 7EH, UK P G J Burney

Correspondence to: Dr H F Guite.

Received 21 September 1995 Returned to autho 24 November 1995

Revised version received 11 April 1996

Accepted for publication

15 April 1996
Keywords: asthma, deaths, accuracy of certification.

To interpret studies of mortality from asthma it is important to know how accurately death certificates identify asthma deaths. Two recent studies have raised the possibility of serious underestimation. Hunt et $a l^{1}$ in the USA found that, when asthma was judged by an expert panel to be the underlying cause of death, it was only recorded as such on 22 of $53(42 \%)$ death certificates. When asthma did appear on the death certificate it was usually an accurate diagnosis ( 22 of 26 cases $(85 \%)$ ). In the UK Goldacre $^{2}$ reported that, of 92 deaths occurring within four weeks of an admission for asthma, only $37 \%$ mentioned asthma in part 1 or part 2 of the death certificate, suggesting under-recording of asthma deaths in the UK.
On the other hand, several studies ${ }^{3-8}$ have reported that UK death certificates wrongly attribute deaths to asthma (false positives). Estimates of the proportion of death certificates that attribute death to asthma which are incorrect lie between $12 \%{ }^{4}$ and $35 \%{ }^{8}$ for all ages. For those under 65 years of age the range is between $13 \%{ }^{8}$ and $24 \%$.

While these studies have comprehensively reviewed all deaths certified as due to asthma, the task would be too large to review deaths from all causes in the populations studied. The search for deaths due to asthma wrongly attributed to another cause (false negatives) has therefore mainly been limited to a review of deaths where asthma was recorded on part 2 of the death certificate. Among UK studies wider searches for false negative death certificates have included a review of deaths certified as due to chronic bronchitis or bronchopneumonia in those under 45 years ${ }^{4}$ or under 55 years, ${ }^{8}$ and a review of deaths from all causes which occurred during a hospital admission for asthma. ${ }^{6}$ Cases of death from asthma were found among these selected deaths from other causes but cannot be used to estimate the full extent of under-recording of asthma on the death certificate.

This study was set up to ascertain the extent to which asthma deaths are wrongly attributed to another cause in the UK.

\section{Methods}

Deaths from all causes were identified in a cohort of people discharged from hospital after treatment for asthma.

\section{IDENTIFICATION OF THE COHORT}

All admissions of patients aged 16-64 years to hospitals within the South East Thames health region in England with a primary diagnosis of asthma (ICD 493) on discharge between 1 April 1989 and 30 September 1990 were identified from the regional inpatient database $(n=$ 3188). In five districts with low rates of clinical coding a review of casualty registers was carried out and revealed a further 104 admissions. The first admission during the study period was designated as the index admission.

\section{IDENTIFICATION OF DEATHS AND CLINICAL} DETAILS

Vital status was ascertained by the National Health Services Central Register (NHSCR) in Southport up to 31 March 1992. Two other sources of deaths in the follow up period were the hospital patient administration systems and 
the yearly regional computerised records of deaths of residents.

Copies of coroners' or hospital post mortem examinations carried out on these patients were obtained. A research assistant was trained to identify relevant sections of the medical notes. The assistant was blind to the cause of death and photocopied the notes from the index admission, the last admission before death or leading to death, and correspondence in the year before death. One of us (HG) photocopied clinical records from the general practitioners' notes in the year before death and discharge summaries from previous inpatient treatment.

THE EXPERT PANEL

The panel consisted of eight experts (four consultants in thoracic and general medicine and four general practitioners with expert knowledge of asthma). The criteria for panel membership were (1) currently practising clinician; (2) involvement in the care of general medical patients (consultants: responsible for "general medical take" as well as the care of patients with chest disease); (3) author of publications on the subject of asthma or have been a national adviser on asthma; (4) agreement to confidentiality regarding hospitals, clinicians, and patients; and (5) within travelling distance of London.

To avoid panel members reviewing notes in detail of patients whose certified cause of death was unlikely to change as a result of an expert opinion, such as deaths from cancer or those for which there was insufficient information at the time of death, one of the authors (HG) screened the notes. Members of the panel briefly checked the excluded notes to ensure that an expert opinion would be unlikely or unable due to lack of information to disagree with the certified cause of death.

The notes of the remaining patients were randomly allocated among the four general practitioners and similarly randomly allocated among the four consultants. Each set of notes was reviewed by one general practitioner and one consultant who made an independent assessment of the underlying cause of death. The general practitioners conferred together on points of fact as did the consultants, but the decision regarding the underlying cause of death was the responsibility of the individual panel member. Disagreements among panel members when assessing the cause of death were resolved by reviewing the notes again in the light of the other panel member's written comments. At this stage panel members were unaware of the identity of the other assessor. Remaining disagreements were discussed in a plenary session. Consensus was defined as agreement between the two original assessors or, in the event of disagreement, between six out of eight panel members.

ETHICAL APPROVAL

Ethical approval was obtained from all ethical committees in the region and permission to photocopy notes was sought from consultants involved in the care of the patient and from the family health service authorities in the region. To preserve confidentiality the patient's name was covered wherever it appeared on the photocopied notes.

\section{STATISTICAL METHODS}

Statistical analysis was carried out using Epi-Info version 6. ${ }^{9}$ The exact binomial distribution was chosen to calculate confidence intervals. The false negative proportion was defined as the proportion of deaths identified as due to asthma by the expert panel which were not certified as asthma. The false positive proportion was defined as the proportion of deaths certified as asthma which the expert panel concluded were not asthma deaths.

\section{Results}

The mean age of the 2382 people in the cohort was 36 years, range 16-64 years; $63 \%$ were women. The mean follow up period was two years and three months (range 18 months to three years). During this time $85(3.6 \%)$ patients were found to have died. The mean age at death was 53 years (median 57 years, range $18-66$ ).

Twenty two patients were excluded from the expert panel review, comprising seven whose underlying cause of death was certified as being due to cancer and 15 for whom there was no clinical information in the three months before death. The panel therefore reviewed 63 deaths from copies of the hospital case notes. In 46 of these $(73 \%)$ notes from primary care in the year before death were available. Post mortem examination records were available in 30 patients $(48 \%)$, but the panel found that few of these included clinical histories and none included histological results to confirm or dispute a diagnosis of asthma. The panel found a further nine cases in whom there was insufficient information to complete a death certificate. There were therefore 54 cases where the panel were confident of the cause of death, plus seven deaths from cancer where the cause of death was undisputed, resulting in 61 of 85 deaths $(72 \%)$ being included in the study.

\section{CAUSE OF DEATH}

Table 1 shows the distribution of the underlying cause of death given on the death certificate compared with the judgement of the expert panel for all deaths in the cohort. Among the 61 cases included in the study 19 recorded asthma as the cause of death on the death certificate (table 2). The panel agreed that asthma was the cause of death in all but one case (case 55 described in appendix A).

The panel identified a further four deaths from asthma. In two of these the underlying cause of death on the death certificate was recorded as chronic obstructive pulmonary disease (cases 7 and 65 described in appendix B) and in two cardiovascular causes (cases 53 and 71 described in appendix C). Overall, four of $22(18 \%, 95 \%$ CI 5 to 40$)$ of all asthma deaths were inaccurately diagnosed as death from another cause (table 2). 
Table 1 Distribution of underlying cause of death recorded on death certificates compared with the expert panel judgement of the underlying cause of death

\begin{tabular}{llcll}
\hline & \multicolumn{2}{l}{$\begin{array}{l}\text { Underlying cause of death determined by the } \\
\text { expert panel }\end{array}$} & \\
\cline { 2 - 4 } Underlying cause of death by death certificate & Asthma & Non-asthma & Not known & Total \\
\hline Asthma & 18 & 1 & 7 & 26 \\
Cystic fibrosis & 0 & 1 & 0 & 1 \\
COPD + other respiratory disorders & 2 & 14 & 5 & 21 \\
CVS (IHD, CVA, PE, DVT) & 2 & 14 & 7 & 23 \\
Lung cancer & 0 & 4 & 0 & 4 \\
Other cancers & 0 & 3 & 0 & 3 \\
Accident/poison/drug/alcohol & 0 & 1 & 3 & 4 \\
Other & 0 & 1 & 1 & 2 \\
Not known & 0 & 0 & 1 & 1 \\
Total & 22 & 39 & 24 & 85 \\
\hline
\end{tabular}

COPD = chronic obstructive pulmonary disease; $\mathrm{CVS}=$ cardiovascular system; IHD = ischaemic heart disease; CVA = cardiovascular accident; $\mathrm{PE}=$ pulmonary embolus; $\mathrm{DVT}=$ deep vein thrombosis.

Table 2 Sensitivity and specificity of underlying cause of death on the death certificate compared with the expert panel judgement

\begin{tabular}{llll}
\hline & \multicolumn{2}{l}{ Expert panel } & \\
\cline { 2 - 3 } Death certificate & Asthma & Not asthma & Total \\
\hline Asthma & 18 & 1 & 19 \\
Not asthma & 4 & 38 & 42 \\
Total & 22 & 39 & 61 \\
\hline
\end{tabular}

False negative $=4 / 22=18 \%(95 \%$ CI $5 \%$ to $40 \%)$

Sensitivity $=18 / 22=82 \%(95 \%$ CI $60 \%$ to $95 \%)$.

False positive $=1 / 19=5 \%(95 \%$ CI $0.1 \%$ to $26 \%)$

Specificity $=38 / 39=97 \%(95 \%$ CI $87 \%$ to $100 \%)$

AGREEMENT BETWEEN EXPERT PANEL MEMBERS

There was initial disagreement on the underlying cause of death between panel general practitioners and consultants in 15 cases; three concerned whether there was sufficient information to determine cause of death, five whether death was due to asthma or COPD, three concerned the appropriateness of pneumonia as an underlying cause of death, and four concerned the extent to which disorder of the cardiovascular system was the underlying cause of death. Nine of these were resolved by change of opinion by one of the experts in the light of further information on the case by the other expert. The other six cases were discussed in a plenary session and complete consensus was reached about the underlying cause of death.
It is noteworthy that, of the four false negative cases (see appendices B and C), in two there was complete agreement initially between the general practitioner and consultant, in case 65 the general practitioner initially thought the patient had COPD but after the plenary discussion agreed with the consensus that the clinical picture was more that of asthma, and in case 71 neither the general practitioner nor consultant thought that death was due to cardiovascular disorder, but initially the consultant thought the disorder was due to COPD. Again, after plenary discussion, he agreed that asthma was a more appropriate diagnosis.

\section{CASES FOR WHICH THERE WAS INADEQUATE}

CLINICAL INFORMATION TO DETERMINE CAUSE OF DEATH

The expert panel were rigorous in excluding cases where there was insufficient information immediately preceding death from which to eliminate death from other causes, such as death by accident or misadventure, as they would in a clinical setting. Twenty four cases were excluded for this reason for 18 of which clinical information was available from the index admission and from the primary care notes. Available clinical details and the certified cause of death of these patients are summarised in table 3 .

\section{Discussion}

This study confirms that in the UK asthma deaths may be misdiagnosed as deaths from cardiovascular disease as well as from chronic obstructive pulmonary disease. This follows the pattern of misattribution found in two other studies ${ }^{110}$ which reviewed deaths from all causes occurring in people with asthma.

The proportion of asthma deaths which were inaccurately diagnosed as death from other causes $(18 \%)$ was similar to the $16 \%$ found to be inaccurately diagnosed in a study in Canada in subjects with milder but persistent asthma (patients receiving at least 10 asthma drug prescriptions in 10 years). ${ }^{10}$ It was, however, much lower than the $58 \%$ false negative rate found in

Table 3 Details of 18 of the 24 patients excluded from the study because of inadequate information immediately before death for whom some clinical information was available

\begin{tabular}{|c|c|c|c|c|c|c|c|}
\hline $\begin{array}{l}\text { Case } \\
\text { no. }\end{array}$ & $\begin{array}{l}\text { Certified cause of } \\
\text { death }\end{array}$ & Age at death & Sex & $\begin{array}{l}\text { Age at onset of } \\
\text { asthma (years) }\end{array}$ & $\begin{array}{l}\text { Min/max recorded } \\
P E F(\text { lmin })\end{array}$ & Smoking history & Further details \\
\hline 13 & Asthma & 48 & $\mathrm{~F}$ & 41 & $150 / 320$ & Never & - \\
\hline 73 & Asthma & 53 & $\mathrm{~F}$ & "Long standing" & $90 / 260$ & Never & - \\
\hline 63 & Asthma & 57 & $\mathrm{~F}$ & NK & $60 / 140$ & Ex 1 year & - \\
\hline 82 & Asthma & 58 & $\mathrm{~F}$ & 22 & $60 / 180$ & Never & - \\
\hline 3 & Asthma & 62 & $M$ & 55 & $110 / 165$ & Ex 5 years & - \\
\hline 89 & Asthma & 64 & M & "As a child" & $80 / 240$ & Never & - \\
\hline 4 & COPD & 52 & $\mathbf{M}$ & 17 & $? / 120$ & Ex 30 years & - \\
\hline 64 & COPD & 61 & $\mathbf{F}$ & NK & $100 / 210$ & Ex 36 years & - \\
\hline 78 & COPD & 63 & $\mathbf{M}$ & NK & $? / 310$ & Ex 8 years & - \\
\hline 57 & COPD & 64 & $\mathbf{M}$ & "As a child" & $70 / 350$ & Ex ? years & - \\
\hline 58 & COPD & 64 & $M$ & NK & $190 / 310$ & Ex 8 years & - \\
\hline 72 & CVS & 48 & $\mathrm{~F}$ & 38 & $200 / 425$ & 5/day & Two previous MIs \\
\hline 76 & CVS & 50 & $\mathbf{M}$ & "As a child" & $200 / 400$ & NK & CABG in past, high $B P$ \\
\hline 2 & CVS & 53 & F & NK & $80 / 290$ & 5/day & Angina, high $\mathrm{BP}$ \\
\hline 5 & CVS & 59 & $\mathbf{M}$ & 55 & NK & $\mathrm{NK}$ & Mitral regurgitation, Churg Strauss syndrome \\
\hline 20 & CVS & 62 & $\mathbf{F}$ & NK & $80 / 360$ & Ex 3 years & $\begin{array}{l}2 \text { weeks before death PEF } 1501 / \mathrm{min} \text {. No } \\
\text { history of heart disease }\end{array}$ \\
\hline 50 & CVS & 63 & $\mathbf{M}$ & 4 & $? / 330$ & Never & Heart failure, high BP \\
\hline 40 & CVA & 66 & $\mathbf{M}$ & 59 & $150 / 260$ & Ex ? years & - \\
\hline
\end{tabular}

$\mathrm{PEF}=$ peak expiratory flow; $\mathrm{MI}=$ myocardial infarction; $\mathrm{CABG}=$ coronary artery bypass graft; $\mathrm{BP}=$ blood pressure; $\mathrm{COPD}=$ chronic obstructive pulmonary disease; CVS = cardiovascular system; CVA = cardiovascular accident; NK = not known 
an American study of non-hospitalised asthmatic subjects (having a medical diagnosis from any source who filled the criteria for a diagnosis of asthma). ${ }^{1}$ Some misattributed deaths may have been missed in our study because of the cases excluded due to lack of information, but among these there are few possible further false negative cases (table 3 ). The difference in the rates between the two studies is probably due to the difference in age of the populations studied, the mean age at death in the study by Hunt et al being 70 years. ${ }^{1}$ Half of the cases misdiagnosed in her study had a death certificate diagnosis of cerebrovascular disease, while only one patient in this study died from cerebrovascular disease. More cases of death from asthma may also have been misdiagnosed in Hunt's study because the patients were non-hospitalised asthmatics and may have been less likely to have a clear diagnosis of asthma when alive. Consideration of asthma as a cause of death would be less likely in those with no previous admissions.

In the UK two previous studies have claimed a net over-recording of asthma deaths ${ }^{57}$ but these were based on limited searches for false negative death certificates. In the light of the findings of our study these claims should be re-examined. Since our study was based on patients who had recently been in hospital with a diagnosis of asthma, the true proportion of false negative death certificates for asthma in the general population is likely to be at least that estimated here or maybe greater.

Post mortem findings should aid the diagnosis of death from asthma but, in general, the panel found the post mortem reports unhelpful. Hunt $e t$ al ${ }^{1}$ also found that the accuracy of diagnosis of asthma as a cause of death was not influenced by the presence of a post mortem report. Post mortem findings can be misleading in the absence of clinical histories since emphysema is found at post mortem examination in most patients who die from status asthmaticus ${ }^{11} 12$ and in about half there is cardiac hypertrophy. ${ }^{11} 12$

The validity of the cause of death based on a retrospective assessment of the notes by clinicians not involved with the case could be questioned. However, the panel members had access to considerable clinical information, including primary care notes in $73 \%$ of cases and details of at least one hospital admission for asthma in all cases. They therefore may have had more information available to them than some of the doctors who signed the death certificates. It is noteworthy that none of the certificates which the panel disagreed with had been signed by general practitioners.

One problem which can arise in the use of expert opinion is that of biased experts. ${ }^{14}$ The experts were chosen according to predetermined criteria to reduce potential bias. One source of bias in this panel may be that, as experts in asthma, they were more likely to diagnose asthma as a cause of death. To reduce the chance of this all consultants on the panel were required to be involved in the care of non-asthmatic patients and general practitioners were included on the panel.
Other problems that can arise in developing a consensus amongst experts is that judgements can be affected by strong individuals within the group or by a convergence to the opinion of the group as a whole..$^{13}$ To avoid this we adapted the Delphi technique for reaching consensus opinion. ${ }^{13} 14$ The essence of this technique is to provide some degree of anonymity to the participants, a chance to revise first responses in the light of others' views, and a process for clarifying the reasons for any differences. Each case was reviewed by a random selection of two experts. This reduced the chance of a general practitioner/ consultant pair converging their opinions over the course of the day. Where there was initial disagreement the general practitioner and consultant reassessed their diagnosis in the light of written comments from the other panel member.

In summary, we have found evidence that in the UK death from asthma may be wrongly certified as death from other causes. The implication for studies of asthma death in this age group is that they should include at least a search for asthma deaths recorded as being due to chronic obstructive pulmonary disease and cardiovascular disease in order to include all cases. An increase in post mortem examinations may not help to improve the accuracy of reporting of asthma deaths unless there is wider use of both clinical history and histological evidence in determining the cause of death.

\section{Appendix A: Certified cause of death asthma but the panel considered death was due to COPD}

Case 55

A man aged 61 years at death who smoked 15-20 cigarettes per day. He had had a right pneumonectomy 10 years ago which was recorded as being due to bronchitis. The only evidence of asthma was a record on the casualty card "known asthmatic past 10 years". Peak flow on index admission was $100 \mathrm{l} / \mathrm{min}$ and was unaltered by salbutamol nebuliser. The highest recorded peak flow was $100 \mathrm{l} / \mathrm{min}$. A post mortem examination carried out reported "the right pleural cavity contained buff-coloured pultaceous material. The left bronchus was congested. Small bronchi on the cut surface of the lungs were thick walled and contained viscid mucus. The parenchyma of the left lung was congested".

\section{Appendix B: Certified cause of death COPD but the expert panel considered death was due to asthma}

Case 7

A 63 year old man, an ex-coal miner and ex-smoker, who had suffered from asthma and eczema since childhood. His lowest peak flow on admission was $120 \mathrm{l} / \mathrm{min}$, the highest recorded was $320 \mathrm{l} / \mathrm{min}$. Six months later he saw his general practitioner because of severe shortness of breath and was admitted to hospital where he died after three days. 
Case 65

A 64 year old man with a history of asthma since the age of 55 years. He had had several admissions for shortness of breath but was well between admissions. He had smoked until the age of 45 years. His lowest peak flow was 110 $1 / \mathrm{min}$ and maximum was $250 \mathrm{l} / \mathrm{min}$. $\mathrm{PCO}_{2}$ on admission was $4.7 \mathrm{kPa}$. He saw his general practitioner seven days before death because of shortness of breath and after admission to hospital he improved. Two days before death he suddenly became very short of breath. He collapsed and was resuscitated briefly after a cardiac arrest call, but further resuscitation was abandoned in view of the prognosis.

\section{Appendix C: Certified cause of death cardiovascular disease but the panel considered death was due to asthma Case 53}

A 46 year old female non-smoker who had suffered from asthma and hayfever for 20 years. On her index admission she was admitted to the ITU after sudden onset of shortness of breath and loss of consciousness. Peak flow reached $450 \mathrm{l} / \mathrm{min}$ by the time of discharge. Diurnal variability in her peak flow recordings at discharge remained high at $62 \%$. Blood pressure was $140 / 100$ and there was no ankle oedema. Three weeks after discharge from this admission she had a sudden severe asthmatic attack and collapsed at home. She died in casualty after an unsuccessful resuscitation attempt. Post mortem findings included a moderately enlarged heart, moderate oedema and congestion in the lungs, moderate chronic bronchitis and emphysema, normal tracheobronchial tree.

Case 71

A 51 year old man who was an ex-smoker who had Buergher's disease resulting in bilateral below knee amputations. On his index admission for asthma he had a peak flow of $350 \mathrm{l} / \mathrm{min}$ which rose to $500 \mathrm{l} / \mathrm{min}$ by discharge. Eight weeks after discharge he was readmitted acutely short of breath, peak flow was recorded as $150 \mathrm{l} / \mathrm{min}$. He improved initially then suddenly deteriorated and remained acutely short of breath until he died four days later. The post mortem findings included hypertrophy of both ventricles and dilatation of the valves; no pulmonary congestion was noted, but copious amounts of purulent secretions were present in the bronchial tree. Extensive bullous emphysema was present.

The members of the expert panel were: $M$ Cochrane, $N$ Eiser A Johnson, D Stableforth (consultants); C Griffiths, M Levy, R McKinley, G Mohan (general practitioners). In addition we would like to thank Tony Fitzgerald for statistical advice, Patrice Beveney, Aruna Dash, and Hilary Barton for their help with the data collection and organisation of the expert panel. The study was funded by the National Asthma Campaign.

1 Hunt LW, Silverstein MD, Reed CE, O'Connell EJ, O'Fallon WM, Yunginger JW. Accuracy of the death certificate in a population-based study of asthmatic patients. $\mathcal{F} A M A$ 1993;269:1947-52.

2 Goldacre MJ. Cause-specific mortality: understanding uncertain tips of the disease iceberg. 7 Epidemiol Community Health 1993;47:491-6.

3 Cochrane GM, Clark TJH. A survey of asthma mortality in patients between ages 35 and 64 in the Greater London patients between ages 35 and 64 in the
hospitals in 1971. Thorax 1975;30: 300-5.

4 Ormerod LP, Stableforth DE. Asthma mortality in Birmingham 1975-7: 53 deaths. BMF 1980;280:687-90.

5 A subcommittee of the BTA research committee. Accuracy of death certificates in bronchial asthma. Accuracy of the certification procedures during the confidential inquiry by the British Thoracic Association. Thorax 1984;39:505-9.

6 Eason J, Markowe HLJ. Controlled investigation of deaths from asthma in hospitals in the North East Thames Region. BM7 1987;294:1255-8.

7 Berrill WT. Is the death rate from asthma exaggerated? Evidence from West Cumbria. BMF 1993;306:193-4

8 Wright SC, Evans AE, Sinnamon DG, MacMahon J Asthma mortality and death certification in Northern Ireland. Thorax 1994;49:141-3.

9 Dean AG, Dean JA, Coulombier D, Brendel KA, Smith DC, Burton AH, et al. Epi-info. Version 6: a word processing, database and statistics program for epidemiology on ing, database and statistics program for epidemiology on microcomputers. Atla

10 Suissa S, Ernst P, Boivin JF, Horwitz RI, Habbick B, Cockroft D, et al. A cohort analysis of excess mortality in asthma and the use of inhaled beta agonists. Am $\mathcal{F}$ Respir Crit Car Med 1994;149:604-10.

11 Bullen SS. Correlation of clinical and autopsy findings in 176 cases of asthma. F Allergy 1952;23:193-203.

12 Messer JW, Peters GA, Bennett WA. Causes of death and pathologic findings in 304 cases of bronchial asthma. Dis Chest 1960;36:616-24

13 Williams PL, Webb C. The Delphi technique: methodological discussion. $\mathcal{F}$ Adv Nursing 1994;19:180-6.

14 Abramson JH. Survey methods in community medicine. Edinburgh: Churchill Livingstone, 1990 . 